\title{
Unusual dual-loop reentry during cavo-tricuspid isthmus-dependent atrial flutter
}

\author{
Satoshi Higuchi ${ }^{1}$ (D) Morio Shoda ${ }^{1} \cdot$ Miwa Kanai $^{1} \cdot$ Kyoichiro Yazaki $^{1} \cdot$ Daigo Yagishita ${ }^{1} \cdot$ Koichiro Ejima $^{1}$. \\ Nobuhisa Hagiwara ${ }^{1}$
}

Received: 12 December 2018 / Accepted: 25 April 2019 / Published online: 14 May 2019

(C) Springer Science+Business Media, LLC, part of Springer Nature 2019

Keywords Atrial flutter $\cdot 3$-dimensional mapping $\cdot$ Dual-loop $\cdot$ Cavo-tricuspid isthmus

A 70-year-old man underwent catheter ablation of symptomatic atrial flutter (AFL). During the AFL, 3-dimensional electroanatomic mapping (RHYTHMIA, Boston Scientific, Natick, MA, USA) demonstrated a dual-loop reentrant propagation, one propagating inside the cavo-tricuspid isthmus (CTI) and the other around the tricuspid annulus in a clockwise direction (Fig. 1). The common pathway of both reentrant circuits was the anterior part of the CTI. Entrainment mapping at a posterior site of the CTI demonstrated a longer post-pacing interval (PPI) than the tachycardia cycle length, suggesting that the intra-isthmus reentrant circuit was not involved in the dominant AFL. Further entrainment mapping at the anterior part of the CTI and along the tricuspid annulus indicated that this tachycardia was a typical clockwise AFL. Radiofrequency applications targeting the anterior CTI alone interrupted the AFL.

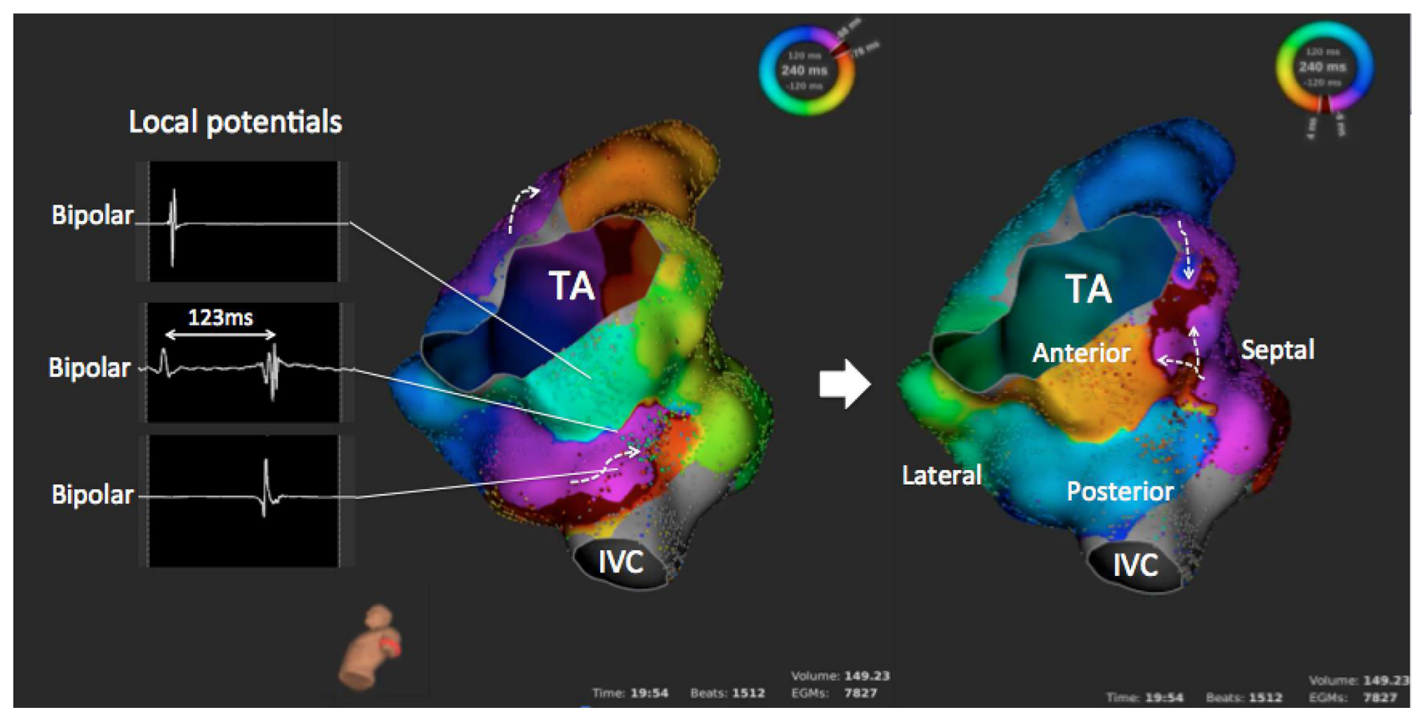

Fig. 1 An endocardial propagation map showing dual-loop reentrant circuits during atrial flutter in the left anterior oblique caudal view. Note that a double potential with an interval of $123 \mathrm{~ms}$ was recorded at the central cavo-tricuspid isthmus. TA tricuspid annulus, IVC inferior vena cava

Morio Shoda

shoda.morio@twmu.ac.jp

1 Department of Cardiology, Tokyo Women's Medical University, 8-1, Kawada-cho, Shinjuku-ku, Tokyo 162-8666, Japan
Ultra-high-density 3D mapping with a high spatial resolution may delineate an unusual double-loop circuit associated with double potentials at the CTI and a long PPI at the posterior part of the CTI.

Publisher's note Springer Nature remains neutral with regard to jurisdictional claims in published maps and institutional affiliations. 